\title{
ThêoH Bias-removal Method ${ }^{\dagger}$
}

\author{
D. A. Howe, J. McGee-Taylor, and T. Tassett \\ National Institute of Standards and Technology \\ 325 Broadway, Boulder, CO 80305 USA
}

\begin{abstract}
A version of Thêo1 variance, called ThêoBR variance (BR for "bias-removed" relative to the Allan variance), is constructed. This relative bias correction is applied over a range of the longest recommended Allan $\tau$ values for a given data run. ThêoH deviation (' $H$ ' to indicate a hybrid combination of ThêoBR and Allan deviations) is the Allan deviation in short term and switches to the ThêoBR deviation in long term. In the presence of non-integer-power-law and mixed noise types, the approach is as effective and less cumbersome than past approaches, and requires little, if any, a priori knowledge or human judgment of data being analyzed. The substantially gained properties of Thêo1 at large $\tau$, to $50 \%$ beyond the longest possible $\tau$ using the Allan deviation alone, can be obtained without Allan bias. Long-term frequency stability can be obtained in essentially onethird less time. For example, a two-month stability can be obtained with three months of data, rather than the four months of data that are usually required for such a point.
\end{abstract}

\section{Introduction}

Frequency stability is characterized by the square-root of the Allan variance. Throughout this paper, 'Avar' is the overlapping Allan variance estimator and 'Adev' is its square root [1]. The maximum-overlap estimator of Theo1 variance is designated Thêo1 variance, and its square root is Thêo1 deviation or just Thêo1-dev. Thêo1 $(\tau, T)$, shown with the circumflex hat $(\hat{\bullet})$, designates the estimator, for a data-run length $T$. This designation is consistent with historic nomenclature ' $\hat{\sigma}_{y}^{2}(\tau, T)$ ' or what is now called Avar, as mentioned, which is the maximum-overlap, unbiased estimator of the Allan variance [1], [2], [3].

Statistical measures such as Adev and Thêo1-dev provide primarily a spectral interpretation of levels and types of relative random clock-frequency instabilities. Theo1 $(\tau)$ variance, where $\tau$ is a sampling time-interval, characterizes an oscillator's frequency domain powerlaw noises of form $S_{y}(f)=f^{\alpha}$ for $-3<\alpha<2$ to exceptionally low $f$. Measures of $S_{y}(f)$ are useful for separating underlying clock noise from other sources of noise in the data run, as well as for providing convenient, practical metrics of frequency instability [4]. The transform to $S_{y}(f)$ from Theo1 variance follows log-log straight lines, similar to the Allan variance [5]. The levels of the Theo1 autocovariance (ACV) functions are close to those of the Allan ACV functions, and for white FM (WHFM) ACV's are identical by definition [6], [7], [8]. The ratio of the translations to $h_{\alpha}$ 's using Theo1 vs. Allan differ as the ratio $1.5^{\alpha}$ for $\tau_{0} \ll \tau$. Thus, Thêo1's

†Contribution of the U. S. Government. Article is not subject to copyright.

1-4244-0074-0/06/\$20.00 @ 2006 IEEE.

1-4244-0074-0/06/\$20.00 C 2006 IEEE.
Allan-bias can be readily removed for common, integer power-law noise, if known, where $\alpha=-2,-1$, and +1 , and $\alpha=+2$, if known to be white phase modulation (WHPM) noise. Two difficulties addressed in this paper are that we often do not confidently know $\alpha$ and it is not necessarily integer over a range of interest of $\tau$-values.

In Section 2, we introduce the concept of a hybrid statistic and its advantages. In Section 3, we define normalized bias as a function of $m$ and $\tau_{0}$ and discuss past ad hoc ways of creating an Allan-unbiased version of Thêo1. In Section 4, we give a direct formula for estimating and removing normalized bias, which acts over a range of $m$ starting with its maximum and obtain what is called ThêoBR variance. ThêoBR variance adjusts Thêo1 variance by a factor computed at the longest $\tau$ values of Avar that have good confidence, namely, up to $20 \%$ of the total data-run time [2]. To obtain the shortest to longest term frequency instability for a given data run, we plot a hybrid statistic, ThêoH deviation as described in Section 5. ThêoH is Adev for small $\tau$, and ThêoBR for large $\tau$. We find that ThêoH reports very long-term frequency stability with excellent confidence at $\tau$-values that are $50 \%$ beyond the longest possible using the Allan deviation alone, even in the presence of mixed noise types. An example is given in Section 6 of an unusual case involving frequency data from a free-running optical oscillator having random changes in what appears as frequency drift at long term.

\section{Hybrid Statistics}

If improved confidence at long-term measurements of frequency stability are important, a 'Total' Allan variance estimator, called Total Avar, has been recommended [2]. Total Avar is Avar that is applied to a simple $\tau$-length extension of the original data [3], [9]. It is an example of a "hybrid" statistic in the sense of improved estimation of frequency stability at long-term $\tau$ values, where the extension is greatest, while essentially computing plain Avar at mid- and short-term $\tau$-values, where the extension becomes progressively smaller [10], [11], [12], [13], [14]. TotalAvar $(\tau, T)$ has dependence on $\tau$ and data-run length $T$ as with Avar and can be regarded as a better estimator of the Allan variance, especially in the presence of frequency drift [15].

In terms of gaining high equivalent degrees of freedom (edf), Thêo1 variance outperforms max-overlap estimators Avar as well as Total Avar, having respective edf's at long $\tau=\frac{T}{2}$ of $2.1,4.3$, and 6 for randomwalk FM (RWFM), flicker FM (FLFM), and white FM (WHFM). With bias relative to the Allan variance re- 
moved, we can thus achieve a time-domain statistical measure that has a six-times increase in edf's, hence, subsantially improved confidence, on the reported level of frequency stability at the longest-term $\tau$-value of Avar for a primary frequency standard properly exhibiting WHFM [16], [17], [18]. Considering this significant gain, we discuss strategies for such bias removal that ultimately lead to ThêoH variance, ' $\mathrm{H}$ ' for a hybrid variance, that can essentially replace Avar and Total Avar.
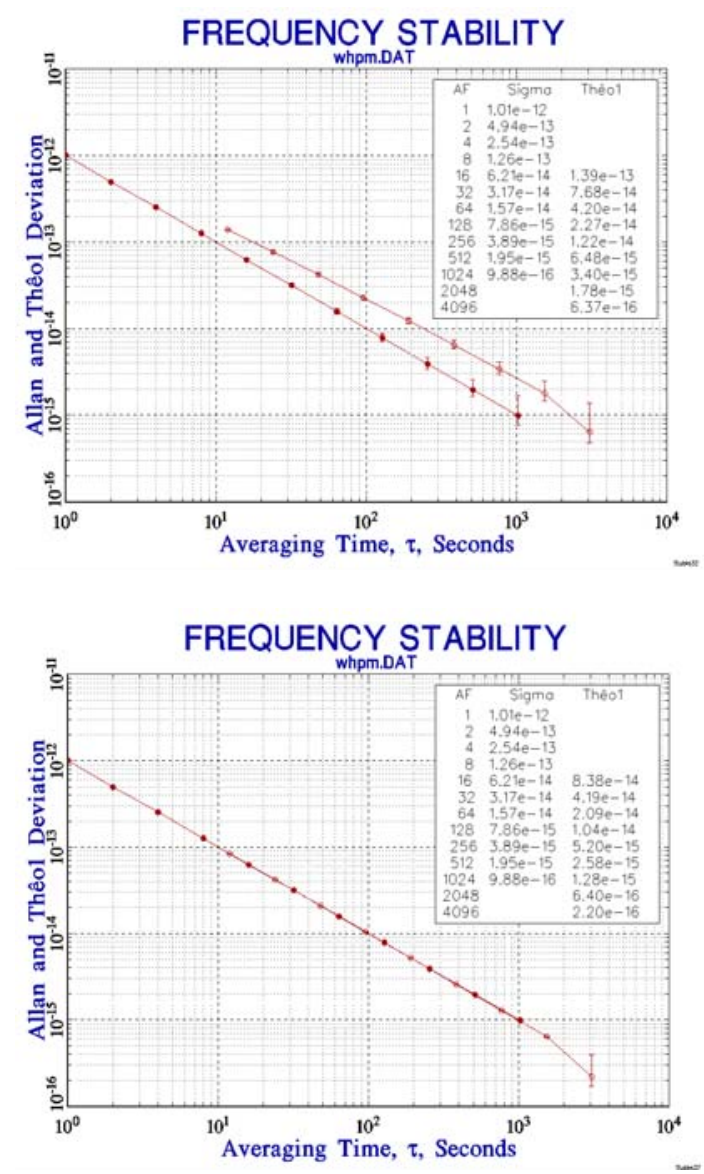

Fig. 1. The upper plot shows Thêo1 and Adev operating on WHPM noise type and the lower plot shows Thêo1 with bias removed relative to Adev using (2) and Allan dead-time bias, or $B_{1}$, function [19], [20].

\section{Methods of Removing Bias Relative to Avar}

The unique data sampling of Theo1 [6], [7], [8] causes bias with respect to zero-dead-time sampling of $\sigma_{y}^{2}(\tau)$. Theo1 $\left(m, \tau_{0}\right)$ relative to the Allan variance can be expressed as a ratio of $\sigma_{y}^{2}(\tau)$ to Theo1 $\left(m, \tau_{0}\right)$ given by a normalized bias as:

$$
\begin{gathered}
\operatorname{nbias}\left(m, \tau_{0}\right) \\
=\frac{\sigma_{y}^{2}(\tau)}{\operatorname{Theo1}\left(m, \tau_{0}\right)}-1 .
\end{gathered}
$$

Thêo1 is defined to be an unbiased estimator of $\sigma_{y}^{2}(\tau)$ in the case of the WHFM noise type. In statistics, for an estimator of a parameter, its bias is defined as the difference between the expectation of the estimator and the true value of the parameter. Bias in the context of this writing means the ratio as given above in (1). An ad hoc method of determining noise type and removing bias previously reported in ref. [6] has since been found to have its bias slightly dependent on $m[7]$. The most accurate dependence is given by the function

$$
\operatorname{nbias}(m)=a+\frac{b}{m^{c}}-1
$$

where $a, b$, and $c$ are empirically derived constants. These constants are summarized for each of the five integer-slope noise types in Table I.

TABLE I

Constant VAlues for the BIAS Functions of ThêO1 Defined in Equation (2).

\begin{tabular}{cccc}
\hline Noise & $\mathrm{a}$ & $\mathrm{b}$ & $\mathrm{c}$ \\
\hline WHPM & 0.09 & 0.74 & 0.40 \\
FLPM & 0.14 & 0.82 & 0.30 \\
WHFM & 1 & 0 & 0 \\
FLFM & 1.87 & -1.05 & 0.79 \\
RWFM & 2.70 & -1.53 & 0.85 \\
\hline
\end{tabular}

A method for determining other noise types is to estimate Avar's own bias, or $B_{1}$, function relative to the standard variance [19], [20]. Unfortunately, Avar bias cannot be defined beyond $\frac{T}{2}$, or half the data run, since Avar is undefined, which leads us to a difficulty in using this method for $\tau>\frac{T}{2}$. Since $B_{1}$ does not exist for the longest $\tau$ values of Thêo1, we cannot determine the noise type and hence correct for Thêo1 bias. Other estimates of noise type are obtainable [21], [22], although use of these methods is limited at this time.

Taken together, the above mentioned methods assume (1) negligible uncertainty of the noise type at a particular value of $m,(2)$ only one noise type occurs, and (3) the noise type fits neatly into one of the five integer-slope noise models.

For these reasons, an estimate of (1) was investigated in regions where essentially all $\tau$-values of Thêo1 and Avar overlap. For a given $\tau_{0}$, uncertainty reduces as the data run increases and the mean-ratio is proportionately weighted toward the longest overlapping $\tau$-values because the number of point estimates in (1) increases with $\tau$. Both of these effects are generally desirable.

\section{Bias-removal Method of ThêoBR}

By use of (1) and starting at the longest Avar $\tau$ values, an unbiased version of Thêo1 variance, called ThêoBR variance (for "Theo bias-removed"), is estimated by

$$
\text { ThêoBR }\left(m, \tau_{0}, N_{x}\right)
$$



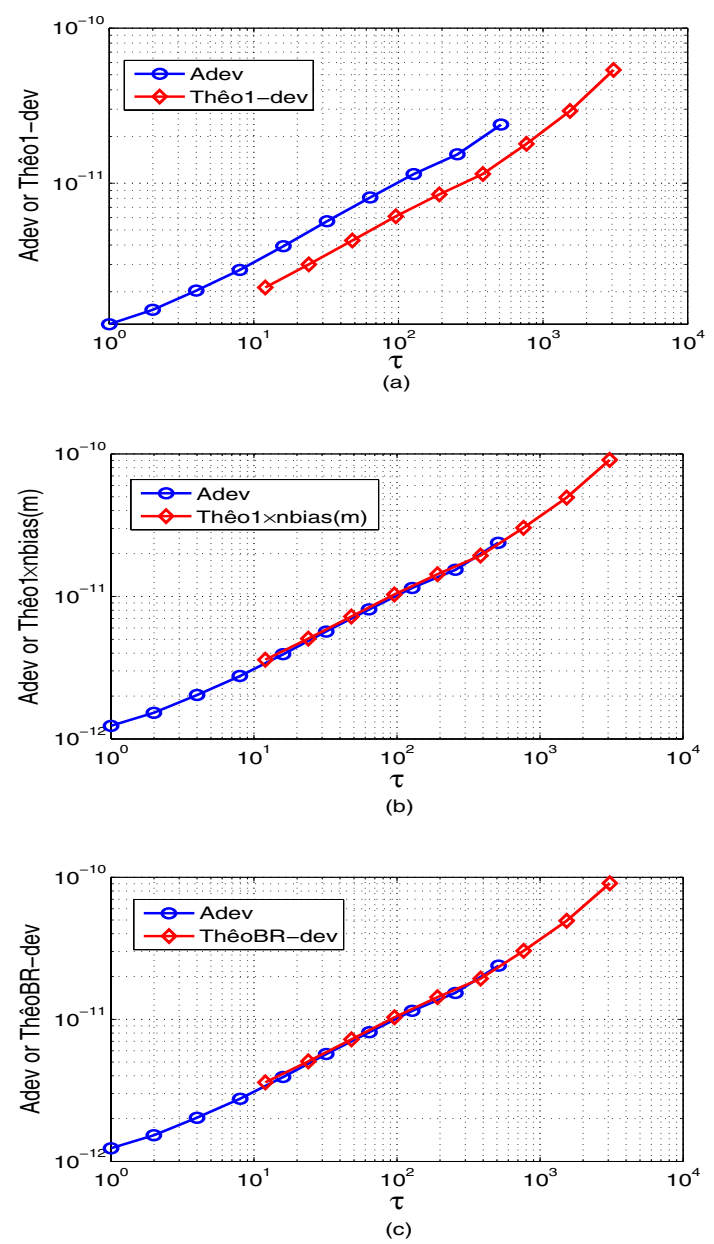

Fig. 2. Shown in (a) is Adev and Theo1-dev for RWFM, while (b) and (c) shows the agreement between using nbias of (1) with Table I vs. TheoBR-dev.

$$
\begin{gathered}
=\left[\frac{1}{n+1} \sum_{i=0}^{n} \frac{\operatorname{Avar}\left(m=9+3 i, \tau_{0}, N_{x}\right)}{\widehat{\operatorname{Theo} 1}\left(m=12+4 i, \tau_{0}, N_{x}\right)}\right] \\
\times \widehat{\operatorname{Theo} 1}\left(m, \tau_{0}, N_{x}\right),
\end{gathered}
$$

where $n=\left\lfloor\frac{0.1 N_{x}}{3}-3\right\rfloor$ (where $\lfloor\cdot\rfloor$ denotes the floor function). Thêo1 is defined in Ref's [6], [7], [8], [23], and Avar has its usual definition [1], [2]. The range in the formula above is based on extensive empirical tests that looked at minimizing bias and uncertainty under the widest range of conditions. Avar is limited to $\tau$ no longer than $20 \%$ of the data-run length as recommended [2] due to nulls in Avar's frequency response that potentially cause excessive variability and downshoots in long term [11], [13]. For $n \geq 0, N_{x} \geq 90$ insures at least one term in the summand for computing a bias of sufficient confidence. The method of determining ThêoBRvariance in (3) does not require a priori assumption of long-term noise type and works with non-integer slope types. Figure 2 shows the agreement between using (1) and Table I vs. TheoBR-dev to obtain an Allanunbiased Thêo1-dev if we in fact know the noise type, which in this case is RWFM. For oscillator noise with multiple types, different biases must be removed that depend on $\tau$. This is unnecessary for short- and midterm $\tau$-values, given that Avar usually has more than adequate confidence for most applications. The advantage of ThêoBR variance is improved confidence at longterm $\tau$ with estimates of frequency stability $50 \%$ beyond that possible with Avar. Rather than compute an everywhere Allan-compatible, bias-removed version of Thêo1, it is more practical to construct a hybrid of Avar and ThêoBR variance.

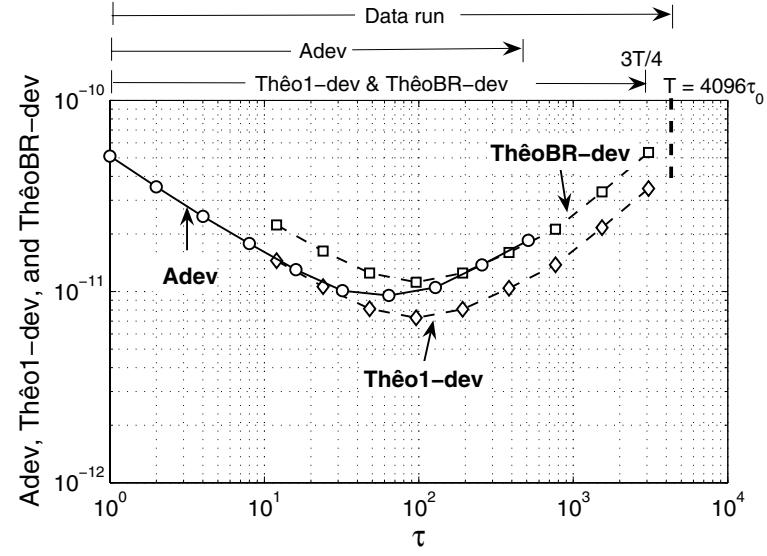

Fig. 3. Adev, Thêo1-dev, and ThêoBR-dev for a data-run time of $T=4096 \tau_{0}$. Data are a mix of WHFM and RWFM noise types. Long-term bias removed (BR) from Thêol-variance is ThêoBRvariance using (3). ThêoBR-dev, like Thêo1-dev, can extend to $\frac{3}{4}$ data-run time.

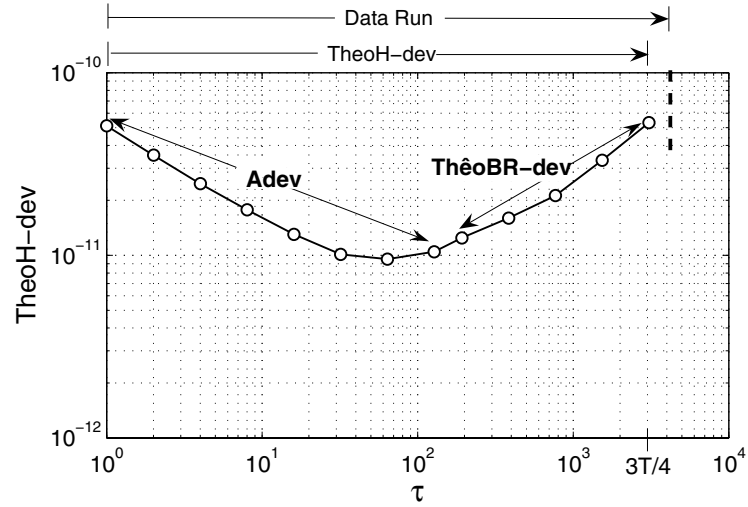

Fig. 4. ThêoH-dev combines Adev and ThêoBR-dev to provide one "Allan-compatible" plot from shortest to longest $\tau$-values.

Figure 3 plots Adev, Thêo1-dev, and ThêoBR-dev for a data run of a mix of WHFM and RWFM noise types whose length $N_{x}=4097$, thus, a data-run time of $T=4096 \tau_{0}$. Note that the effect of bias removal from Thêo1-variance using (3) results in ThêoBR-dev properly resuming where Adev leaves off. ThêoBR-dev, like Thêo1-dev, can extend to $\frac{3}{4}$ data-run length and is not 
subject to downshoots [23].

\section{Definition of ThêoH}

Define a hybrid frequency stability estimator called ThêoH as a composite of $\operatorname{Avar}\left(m, \tau_{0}, N_{x}\right)$ and TheoBR $\left(m, \tau_{0}, N_{x}\right)$, namely,

$$
\begin{gathered}
\operatorname{ThêoH}\left(m, \tau_{0}, N_{x}\right) \\
= \begin{cases}\operatorname{Avar}\left(m, \tau_{0}, N_{x}\right), & \text { for } 1 \leq m<\frac{k}{\tau_{0}} \\
\operatorname{TheoBR}\left(m, \tau_{0}, N_{x}\right), & \text { for } \frac{k}{0.75 \tau_{0}} \leq m \leq N_{x}-1, \\
\text { m even }\end{cases}
\end{gathered}
$$

where $k$ is the largest $\tau \leq 10 \% T$ where $\operatorname{Avar}\left(m, \tau_{0}, N_{x}\right)$ has sufficient confidence. ThêoH is $\operatorname{Avar}\left(m, k, N_{x}\right)$ up to $10 \%$ of the length of the data run and $\widehat{\operatorname{TheoBR}}\left(m, k, N_{x}\right)$ thereafter. As Avar's statistical properties become poorer and poorer in long term, we switch to ThêoBR variance, which has twice the range of $m$-values than Avar and different dependence on $m$ of $\tau=0.75 m \tau_{0}$ and $\tau=m \tau_{0}$, respectively. Figure 4 shows an example of usage of the hybrid statistic by applying the recipe in (4) to obtain ThêoH deviation for the same data used in Figure 3. Slight variations in where the switch occurs from Avar to ThêoBR variance have essentially negligible effect since overlapping $\tau$-values are closely matched anyway.
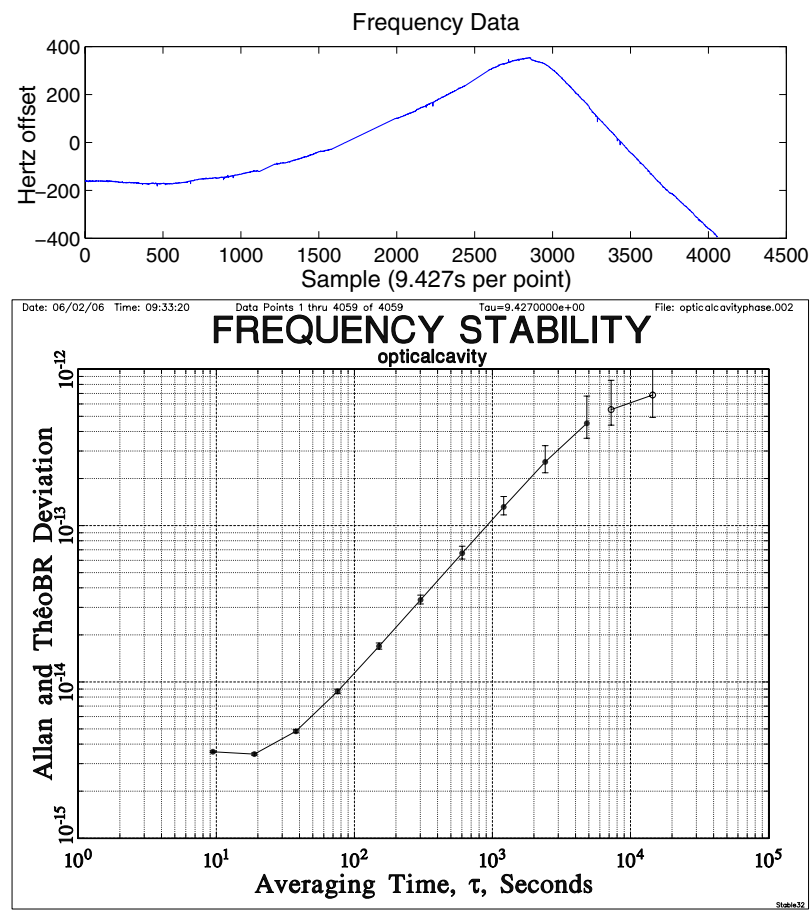

Fig. 5. Allan-compatible plot from shortest to longest-possible $\tau$ values of a laser frequency that is locked to a stable optical cavity. Data are courtesy of J. Bergquist and S. Diddams, June, 2006.

\section{Beyond Avar}

ThêoBR variance can be viewed as calculating the expectation value of Avar at sample times longer than the last possible $\tau$-value of Avar, although this is not the way that "expectation value" is usually meant. This is useful for determining ways of removing frequency drift, given that it is unreliable to extrapolate any timedomain statistic into its unobservable long- $\tau$ region [24] for such purposes. It is important to emphasize that the points computed using ThêoBR $\left(m, k, N_{x}\right)$ in (3) are based on a new class of long-stride statistics and are not an extrapolation of Avar. To show that this is true, studies using a wide range of simulated and actual clock data [6] show that, consistent with confidence intervals, ThêoBR variance calculates the same long-term frequency stability that Avar would otherwise calculate, given more data. Tests of short segments of very long data runs are a way to assess how well ThêoBR variance anticipates the value that Avar would report as increasingly longer segments of a very long data run are used. These studies included steep, mixed integer, and noninteger noise types that change beyond the longest $\tau$ of Avar [8].

Tested software [25] executes ThêoH and other dataanalysis tools. Online MatLab code is available also [26]. Figure 5 shows recent frequency stability results of a free-running $563 \mathrm{~nm}$ laser that is ultimately locked to a mechanically stable optical cavity at approximately 2.80 $\mathrm{x} 10^{14} \mathrm{~Hz}$ with methodology and measurement described in [27]. This is an interesting illustration of exceptional short-term frequency stability in the presence of steep $\tau^{+1}$ slope that might be interpreted as frequency drift. However, systematic drift is not indicated in the raw frequency data shown at the top of Figure 5. Indeed, ThêoBR deviation is computed at the longest two $\tau$ values at $\sim 7,000 s$ and $\sim 14,000 s$ and indicates a long-term slope change toward apparent RWFM whose level can be estimated, even for this limited data run.

Noteworthy is that frequency-domain $S_{y}(f)$ can be estimated to extremely low $f$ using ThêoH variance. Time-to-frequency domain coefficients that have traditionally been used with Avar [1], [2] can continue to be used with ThêoH variance. An important benefit is that, at long term, ThêoH deviation has enough narrowness in its range of noise-slope types as compared to Adev that it enables faster detection of the onset of nonstationary noise types of FLFM or RWFM [28], [29].

\section{Conclusion}

We have formulated ThêoH deviation that consists of Adev for short and mid $\tau$-values in combination with points at very long $\tau$-values of Thêo1 with bias removed (BR) in such a way that mixed, non-integer noise types can be accurately characterized for evaluations that extend to $50 \%$ longer than possible using Adev alone. ' $\mathrm{H}$ ' of ThêoH is used to indicate that it is a $\underline{h}$ ybrid statistic of Adev in short term and ThêoBR ( $\underline{b}$ ias $\underline{r}$ emoved) de- 
viation in long term. Thêo1 variance is the estimator of a new class of theoretical variance that averages every permissible squared second-difference of time-error sampling intervals in a data run.

ThêoBR deviation provides (1) automatic correction for bias relative to Adev, even for non-integer power-law noises, (2) improved power-law noise determination at very long $\tau$-values when used in combination with Adev, namely, using ThêoH-vs.- $\tau$ slopes, (3) characterization to longer $\tau$-values than with Adev, and (4) $S_{y}(f)$ to extremely low $f$ using existing Allan time-to-frequency domain translation coefficients.

\section{References}

[1] D. B. Sullivan, D. W. Allan, D. A. Howe, and F. L. Walls (Editors), Characterization of Clocks and Oscillators, Natl. Inst. Stand. Technol. Technical Note 1337, 1990.

[2] "IEEE Std 1139-1999: Standard Definitions of Physical Quantities for Fundamental Frequency and Time MetrologyRandom Instabilities," IEEE-SA Standards Board, J.R. Vig, chairperson, March, 1999.

[3] D. A. Howe, "An Extension of the Allan Variance with Increased Confidence at Long Term," Proc. 1995 IEEE International Frequency Control Symposium, May 31-June2, San Francisco, CA, USA, pp. 321-329, 1995.

[4] J.A. Barnes, A.R. Chi, L.S. Cutler, D.J. Healy, D.B. Leeson, T.E. McGunigal, J.A. Mullen, Jr., W.L. Smith, R.L. Sydnor, R.F.C Vessot, G.M.R. Winkler, "Characterization of frequency stability," IEEE Trans. Instrum. Meas., IM-20, pp. 105-120, 1971.

[5] D.W. Allan, "Statistics of Atomic Frequency Standards," Proc. IEEE 54, pp. 221-230, Feb., 1966.

[6] D. A. Howe and T. K. Peppler, "Very Long-term Frequency Stability: Estimation using a Special-purpose Statistic", Proc. 2003 Joint Mtg. IEEE Intl. Freq Cont. Symp and EFTF Conf, pp. 233-238, May, 2003.

[7] D. A. Howe and T. N. Tasset, "Theo1: Characterization of Very Long-term Frequency Stability", Proc. 18th European Frequency and Time Forum, April, 2004.

[8] D. A. Howe, "ThêoH: a hybrid, high-confidence statistic that improves on the Allan deviation," Metrologia, accepted for publication, 2006.

[9] D. A. Howe, "Methods of Improving the Estimation of Longterm Frequency Variance," Proc. European Frequency and Time Forum, pp. 91-99, 1997.

[10] D. A. Howe and C. A. Greenhall, "Total variance: a progress report on a new frequency stability characterization," Proc. 29th Ann. Precise Time and Time Interval Meeting, pp. 39$48,1997$.

[11] C. A. Greenhall, D. A. Howe and D. B. Percival, "Total Variance, an Estimator of Long-Term Frequency Stability," IEEE Trans. Ultrasonics, Ferroelectrics, and Freq. Control, UFFC46, no. 5, pp. 1183-1191, Sept., 1999.

[12] D. A. Howe, "Total Variance Explained," Proc. 13th European Frequency and Time Forum and 1999 IEEE International Frequency Control Symposium, pp. 1093-1099, April, 1999.

[13] D. A. Howe, "Total Deviation Approach to Long-Term Characterization of Frequency Stability," IEEE Trans. Ultrasonics, Ferroelectrics, and Freq. Control, UFFC-47 no. 5, pp. 11021110, Sept., 2000.

[14] D. A. Howe and T. Peppler, "Definitions of "total" estimators of common time-domain variances," Proc. 2001 IEEE International Frequency Control Symposium, pp. 127-132, June, 2001.

[15] D. A. Howe and K. J. Lainson, "Effect of Drift on TOTALDEV," Proc. 1996 IEEE International Frequency Control Symposium, pp. 883-889, 1996.

[16] T. E. Parker, S. R. Jefferts, T. P. Heavner, and E. A. Donley, "Operation of the NIST-F1 caesium fountain primary frequency standard with a maser ensemble, including the im- pact of frequency transfer noise," Metrologia, 42, pp. 423-430, Sept., 2005.

[17] A. Godone, S. Micalizio, C. E. Claudio, and F. Levi, "The Pulsed Rubidium Clock," IEEE Trans. Ultrasonics, Ferroelectrics, and Freq. Control, UFFC-53 no. 3, pp. 525-529, March, 2006.

[18] L.Sojdr, J.Cermak, and G.Brida, "Comparison of HighPrecision Frequency-Stability Measurement Systems," Proc. 2004 IEEE International FCS, pp. 317-325, May, 2003.

[19] J.A. Barnes, "Tables of Bias Functions, $B_{1}$ and $B_{2}$, for Variances Based on Finite Samples of Processes with Power Law Spectral Densities," Nat. Bur. Stands. (U.S.) Technical Note, TN-375, January, 1969.

[20] D.A. Howe, R.L. Beard, C.A. Greenhall, F. Vernotte, W.J. Riley, and T.K. Peppler, "Enhancements to GPS Operations and Clock Evaluations using a Total Hadamard Deviation," IEEE Trans. Ultrasonics, Ferroelectrics, and Freq. Control, UFFC-52, no. 8, August, 2005.

[21] W.J. Riley and C.A. Greenhall, "Power Law Noise Identification Using the Lag 1 Autocorrelation," Proc. 18th European Frequency and Time Forum, April, 2004.

[22] C.A. Greenhall and W.J. Riley, "Uncertainty of Stability Variances based on Finite Differences," Proc. 35th Ann. Precise Time and Time Interval Meeting, pp. 267-279, 2003.

[23] T. N. Tasset, D.A. Howe, and D.B. Percival, "Theol confidence intervals," Proc. 2004 IEEE International FCS, pp. 725-728, Aug., 2004.

[24] C. A. Greenhall, "A Frequency-Drift Estimator and Its Removal from Modified Allan Variance," Proc. 1997 IEEE International Frequency Control Symposium, pp. 428-432, May, 1997.

[25] Stable 32, Frequency Stability Analysis for Windows and NT, Hamilton Technical Services, 650 Distant Island Drive, Beaufort, SC 29907 USA, Phone: 843-525-6495, http://www.wriley.com/. No endorsement is implied.

[26] Private communications with Ken Senior, Naval Research Laboratory, Ken.Senior@nrl.navy.mil. MatLab is a registered trademark of MathWorks, Inc. No endorsement is implied. Shared code is subject to change and is a cooperative service for users. Any such sharing is expressly exempt from any liability.

[27] B. C. Young, F. C. Cruz, W. M. Itano, and J. C. Bergquist, "Visible Lasers with Subhertz Linewidths," Phys. Rev. Lett., 82, no. 19, pp. 3799-3802, May, 1999.

[28] J.A. McGee and D.A. Howe, "TheoH and Allan Deviation as Power-Law Noise Estimators," accepted and in progress to IEEE Trans. Ultrasonics, Ferroelectrics, and Freq. Control, 2006.

[29] C. R. Ekstrom and P. A. Koppang, "Error Bars for ThreeCornered Hats," IEEE Trans. Ultrasonics, Ferroelectrics, and Freq. Control, UFFC-53, no. 5, pp. 876-879, May, 2006. 